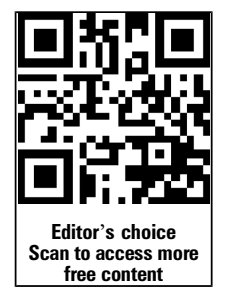

\title{
Role of late gadolinium enhancement cardiovascular magnetic resonance in the risk stratification of hypertrophic cardiomyopathy
}

\author{
Tevfik F Ismail, ${ }^{1,2}$ Andrew Jabbour, ${ }^{1,2}$ Ankur Gulati, ${ }^{1}$ Amy Mallorie, ${ }^{1,2}$ Sadaf Raza, ${ }^{1,2}$ \\ Thomas E Cowling, 1,2 Bibek Das, 1,2 Jahanzaib Khwaja, 1,2 Francisco D Alpendurada, ${ }^{1}$ \\ Ricardo Wage, ${ }^{1}$ Michael Roughton, ${ }^{3}$ William J McKenna, ${ }^{4}$ James C Moon, ${ }^{4}$ \\ Amanda Varnava, ${ }^{5}$ Carl Shakespeare, ${ }^{1,6}$ Martin R Cowie, ${ }^{1,2}$ Stuart A Cook, ${ }^{1,2}$ \\ Perry Elliott, ${ }^{4}$ Rory O'Hanlon, ${ }^{1}$ Dudley J Pennell, ${ }^{1,2}$ Sanjay K Prasad ${ }^{1,2}$
}

\begin{abstract}
- Additional material is published online only. To view please visit the journal online (http://dx.doi.org/10.1136/ heartjnl-2013-305471).

${ }^{1}$ Cardiovascular Magnetic Resonance Unit, Royal Brompton Hospital, London, UK

${ }^{2}$ Imperial College London, London, UK

${ }^{3}$ R-Squared Statistics, London, UK

${ }^{4}$ Institute of Cardiovascular Science, University College London, London, UK ${ }^{5}$ West Hertfordshire Hospitals NHS Trust, Hertfordshire, UK ${ }^{6}$ Department of Cardiology, South London Healthcare NHS Trust, London, UK
\end{abstract}

Correspondence to Professor Dudley J Pennell, Cardiovascular Magnetic Resonance Unit,

Royal Brompton Hospital, Sydney Street,

London SW3 6NP, UK: d.pennell@ic.ac.uk

Received 27 January 2014 Revised 26 May 2014 Accepted 1 June 2014 Published Online First 24 June 2014

\section{SLinked}

- http://dx.doi.org/10.1136/ heartinl-2014-306295

- http://dx.doi.org/10.1136/ heartjnl-2014-306762

\section{CrossMark}

To cite: Ismail TF,

Jabbour A, Gulati A, et al. Heart 2014; 100 :

1851-1858.

\section{ABSTRACT}

Objective Myocardial fibrosis identified by late gadolinium enhancement (LGE) cardiovascular magnetic resonance (CMR) in patients with hypertrophic cardiomyopathy (HCM) is associated with adverse cardiovascular events, but its value as an independent risk factor for sudden cardiac death (SCD) is unknown. We investigated the role of $L G E-C M R$ in the risk stratification of HCM.

Methods We conducted a prospective cohort study in a tertiary referral centre. Consecutive patients with HCM $(n=711$, median age 56.3 years, IQR 46.7-66.6; 70.0\% male) underwent LGE-CMR and were followed for a median 3.5 years. The primary end point was SCD or aborted SCD.

Results Overall, 471 patients (66.2\%) had myocardial fibrosis (median 5.9\% of left ventricular mass, IQR: 2.2-13.3). Twenty-two (3.1\%) reached the primary end point. The extent but not the presence of fibrosis was a significant univariable predictor of the primary end point (HR per 5\% LGE: $1.24,95 \% \mathrm{Cl} 1.06$ to $1.45 ; p=0.007$ and HR for LGE: $2.69,95 \% \mathrm{Cl} 0.91$ to $7.97 ; p=0.073$, respectively). However, on multivariable analysis, only LV-EF remained statistically significant (HR: 0.92, 95\% $\mathrm{Cl} 0.89$ to $0.95 ; \mathrm{p}<0.001)$. For the secondary outcome of cardiovascular mortality/aborted SCD, the presence and the amount of fibrosis were significant predictors on univariable but not multivariable analysis after adjusting for LV-EF and non-sustained ventricular tachycardia.

Conclusions The amount of myocardial fibrosis was a strong univariable predictor of SCD risk. However, this effect was not maintained after adjusting for LV-EF. Further work is required to elucidate the interrelationship between fibrosis and traditional predictors of outcome in HCM.

\section{INTRODUCTION}

Hypertrophic cardiomyopathy (HCM) is a common myocardial disorder that in a minority of patients is associated with sudden cardiac death (SCD). ${ }^{1}$ Data from observational studies suggest that implantable cardioverter-defibrillator (ICD) therapy effectively prevents $\mathrm{SCD},{ }^{2}$ but also shows that the majority of ICD recipients do not receive appropriate therapies and have a high incidence of device-related complications. ${ }^{3}$ There is, therefore, a need to improve the identification of patients at risk. Current approaches to risk stratification rely on the assessment of a small number of clinical features that have been shown in observational cohort studies to associate with a greater risk of SCD. ${ }^{4}$ In aggregate, these clinical risk markers are associated with a substantially increased incidence of SCD in long-term studies, but are poorly predictive when they occur in isolation. ${ }^{4-6}$

Myocardial fibrosis has been implicated in the pathogenesis of SCD in HCM. ${ }^{1} 7$ There has therefore been considerable interest in the use of late gadolinium enhancement (LGE) cardiovascular magnetic resonance (CMR) to detect and quantify myocardial fibrosis in vivo. ${ }^{8} 9$ Initial prospective cohort studies with short-term follow-up and therefore low event rates have suggested that the presence and amount of fibrosis as detected by LGE-CMR is of potential prognostic importance. ${ }^{9} 10$ These studies focused on surrogate end points, ${ }^{8} 11$ and despite the use of broad composite outcome measures, were inadequately powered to address SCD risk or to adjust for the impact of confounding variables. $^{12-15}$ Thus, the role of LGE-CMR in the risk stratification of HCM for SCD and cardiovascular mortality remains unresolved. ${ }^{9} 1016$ We therefore sought to determine the independent prognostic significance of myocardial fibrosis in a large consecutive HCM cohort with long-term follow-up.

\section{METHODS}

\section{Study population}

From September 2000 to June 2011, 815 consecutive patients with HCM referred for CMR at Royal Brompton Hospital were considered for enrolment. Reasons for referral included diagnostic evaluation, confirmation of diagnosis, family screening and assessment of disease severity. HCM was diagnosed in accordance with standard clinical guidelines. ${ }^{17}$ Patients were excluded if they had previous myectomy or alcohol septal ablation $(n=21)$; previous myocardial infarction $(n=14)$; or contraindications to CMR and gadolinium-based contrast agents $(n=12)$. Eight patients withdrew consent for study participation and 49 were lost to follow-up, resulting in a final study cohort of 711 patients. The study was performed in accordance with the Declaration of Helsinki and was approved by the National 
Research Ethics Service, with written informed consent obtained from all patients.

\section{Image acquisition}

Images were acquired using $1.5 \mathrm{~T}$ scanners (Avanto/Sonata, Siemens, Erlangen, Germany) with full myocardial coverage. Balanced steady-state free-precession sequences were used to obtain breath-hold cine images in three long-axis planes, followed by a contiguous stack of short-axis slices from the atrioventricular ring to the apex. ${ }^{18}$ Late enhancement images were acquired $\sim 10 \mathrm{~min}$ after the administration of $0.1 \mathrm{mmol} / \mathrm{kg}$ intravenous gadolinium contrast agent (gadopentetate dimeglumine/ gadobutrol, Bayer, Berlin, Germany) with an inversion recoveryprepared gradient-echo sequence. ${ }^{19}$ Inversion times were optimised to null normal myocardium with images acquired in two orthogonal phase-encoding directions to exclude artefact.

\section{Image analysis}

LV volumes, function, mass and EF were measured using a semiautomated threshold-based technique (CMRtools, Cardiovascular Imaging Solutions, London, UK). All volume and mass measurements were indexed to body surface area. Late enhancement was defined as an area of high signal intensity on a background of adequately nulled myocardium present in two orthogonal phase-encoding directions. The amount of LGE was quantified from a short-axis stack of images with dedicated software using the full-width at half-maximum method (CMR42, Circle Cardiovascular Imaging, Calgary, Canada). ${ }^{12} 2021$ This was expressed as present or absent, and its amount was quantified as a percentage of LV mass as previously described. ${ }^{15} 2223$

\section{Definition of outcome events and follow-up}

All events were adjudicated by the consensus of an independent end point committee blinded to the CMR results. The primary end point was SCD or aborted SCD. The secondary end points were: a composite of cardiovascular mortality (defined as death due to heart failure, SCD, or non-haemorrhagic stroke), aborted SCD or cardiac transplantation; and all-cause mortality.

SCD was defined as unexpected death either within $1 \mathrm{~h}$ of cardiac symptoms in the absence of progressive cardiac deterioration; during sleep; or within $24 \mathrm{~h}$ of last being seen alive. ${ }^{24}$ Heart failure death was defined as death associated with unstable progressive deterioration of pump function despite active therapy. Aborted SCD was diagnosed in patients who received an appropriate ICD discharge for ventricular arrhythmia or had a non-fatal episode of ventricular fibrillation (VF) or spontaneous sustained ventricular tachycardia (VT) (>30 s in duration) causing haemodynamic compromise and/or requiring cardioversion.

Survival status was determined through the UK National Strategic Tracing Service. The date and cause of death were determined using a combination of death certificates; postmortem data when available; communication with patients' primary care physicians and cardiologists; and hospital records. All patients were evaluated for non-fatal events using a standardised telephone and/or postal questionnaire. The patients' primary care physicians and cardiologists were contacted to verify questionnaires and to obtain details of potential aborted SCD events, including the results of relevant ICD interrogations.

\section{Statistical analysis}

Continuous variables are expressed as mean \pm SD for normally distributed variables and as medians with IQR for non- parametric data. Differences between parametric continuous variables were assessed using Student $t$ tests, and for nonparametric data, the Mann-Whitney U test. Categorical data are presented as frequencies and percentages, with differences assessed using the $\chi^{2}$ and Fisher's exact tests as appropriate. The start of follow-up was defined as the date of initial CMR evaluation. Patients were censored at the time of their last clinical follow-up. For composite end points, only the first event in each patient was considered for analysis. Univariable cumulative survival analysis was performed using the Kaplan-Meier method with a log-rank test to assess the significance of any difference between groups. To adjust for potential confounders, multivariable Cox proportional hazards modelling was carried out using covariates identified as significant on univariable analysis or based on previous clinical experience or models. Survival models were assessed for collinearity; interactions between covariates; and to ensure validity of the proportional hazards assumption. The number of covariates examined was restricted to maintain $\geq 10$ events per degree of freedom. Two-tailed values of $\mathrm{p}<0.05$ were considered statistically significant. Statistical analysis was performed using Stata SE V.12.0 (StataCorp, College Station, Texas, USA).

\section{RESULTS}

\section{Study population}

The baseline demographic and clinical characteristics of the study population are summarised in table 1 . The median duration of follow-up was 3.5 years (IQR: 2.1 years to 5.6 years) amounting to 2852 patient-years of follow-up. The median age at study entry was 56.3 years. The majority of patients were male (70.0\%) and minimally symptomatic $(92.7 \%$ New York Heart Association (NYHA) class I/II). Patients with fibrosis were more likely to have extreme hypertrophy (LV wall thickness $\geq 30 \mathrm{~mm}$ ) and non-sustained VT at baseline, giving rise to a greater proportion with two or more risk factors for SCD.

\section{CMR findings}

Patients with fibrosis had more severe hypertrophy compared with those without fibrosis exhibiting significantly higher maximum LV wall thickness and indexed LV mass (table 2). At baseline, LV-EF was significantly lower in those with fibrosis than those without.

Figure 1 illustrates some representative examples of patterns and severity of fibrosis seen in the study cohort. Two-thirds of the cohort exhibited LGE (66.2\%) with a median amount of 5.9\% of LV mass (IQR: 2.2 to 13.3). A maximum end-diastolic wall thickness of $\geq 19 \mathrm{~mm}$ was found on receiver operator characteristic analysis to optimally predict the presence of LGE with a sensitivity of $65.0 \%$, specificity of $77.5 \%$ and Harrell's cstatistic of 0.71 (95\% CI: 0.68 to 0.75 ). The positive and negative predictive values were $85.0 \%$ and $53.0 \%$, respectively, in our cohort. For patients with LV-EF $\leq 55 \%$, the median amount of LGE was $8.0 \%$ of LV mass (IQR 2.9 to 31.4 ).

\section{Primary outcome}

During follow-up, $22(3.1 \%)$ of the 711 patients reached the primary outcome measure of SCD or aborted SCD (table 3). Overall, 18 (3.8\%) patients in the fibrosis group versus $4(1.7 \%)$ in the no fibrosis group reached the primary outcome (HR LGE: $2.69,95 \%$ CI 0.91 to $7.97, p=0.073$ ) (figure 2). On univariable analysis, the amount of fibrosis was a significant predictor of outcome (HR per 5\% LGE 1.24, 95\% CI 1.06 to $1.45, \mathrm{p}=0.007)$. A previous history of sustained VT/VF (HR: 7.76, 95\% CI 1.79 to 33.57 ; $\mathrm{p}=0.006$ ), and LV-EF (HR: 0.92, 
Table 1 Baseline clinical and demographic characteristics of the study cohort

\begin{tabular}{|c|c|c|c|c|}
\hline & No fibrosis & Fibrosis & All patients & p Value \\
\hline Characteristic-n (\%) & $240(33.8)$ & $471(66.2)$ & 711 & \\
\hline Median follow-up-years (IQR) & $4.0(2.9,5.8)$ & $3.2(1.9,5.5)$ & $3.5(2.1,5.6)$ & $<0.001$ \\
\hline Median age at study entry-years (IQR) & $56.0(46.1,66.4)$ & $56.9(47.1,66.8)$ & $56.3(46.7,66.6)$ & 0.686 \\
\hline Median age at diagnosis-years (IQR) & $55.7(45.5,66.2)$ & $54.7(45.9,65.5)$ & $55.0(45.8,65.6)$ & 0.742 \\
\hline Male sex-n (\%) & $164(68.3)$ & $334(70.9)$ & $498(70.0)$ & 0.478 \\
\hline Atrial fibrillation-n (\%) & $3(1.3)$ & $19(4.2)$ & $22(3.2)$ & 0.045 \\
\hline Apical phenotype-n (\%) & $60(25.5)$ & $66(14.2)$ & $126(18.0)$ & $<0.001$ \\
\hline \multicolumn{5}{|l|}{ Risk factors for SCD } \\
\hline Sustained VT/VF-n (\%) & $3(1.3)$ & $11(2.4)$ & $14(2.0)$ & 0.326 \\
\hline Family history of SCD—n (\%) & $25(10.5)$ & $51(10.9)$ & $76(10.8)$ & 0.859 \\
\hline LV wall thickness $\geq 30 \mathrm{~mm}-\mathrm{n}(\%)$ & $0(0)$ & $32(6.8)$ & $32(4.5)$ & $<0.001$ \\
\hline Resting LVOT obstruction $\geq 30 \mathrm{~mm} \mathrm{Hg}-\mathrm{n}(\%)$ & $65(27.1)$ & $143(30.4)$ & $208(29.3)$ & 0.364 \\
\hline Non-sustained VT-n (\%) & $4(1.7)$ & $34(7.3)$ & $38(5.4)$ & 0.002 \\
\hline Unexplained syncope-n (\%) & $19(8.1)$ & $51(11.0)$ & $70(10.0)$ & 0.220 \\
\hline \multicolumn{5}{|l|}{ Number of risk factors for SCD-n $(\%)^{*}$} \\
\hline 0 & $142(59.2)$ & $232(49.3)$ & $374(52.6)$ & 0.005 \\
\hline 1 & $81(33.8)$ & $170(36.1)$ & $251(35.3)$ & \\
\hline $2+$ & $17(7.1)$ & $69(14.7)$ & $86(12.1)$ & \\
\hline \multicolumn{5}{|l|}{ NYHA functional class-n (\%) } \\
\hline I & $157(66.5)$ & $282(60.8)$ & $439(62.7)$ & 0.331 \\
\hline II & $65(27.5)$ & $145(31.3)$ & $210(30.0)$ & \\
\hline III & $12(5.1)$ & $35(7.5)$ & $47(6.7)$ & \\
\hline IV & $2(0.9)$ & $2(0.4)$ & $4(0.6)$ & \\
\hline \multicolumn{5}{|l|}{ Medications at baseline $-\mathrm{n}(\%)$} \\
\hline$\beta$-blocker & $69(30.9)$ & $203(46.1)$ & $272(41.0)$ & $<0.001$ \\
\hline $\mathrm{Ca}^{2+}$-channel blocker & $30(14.1)$ & 79 (19.6) & $109(17.7)$ & 0.088 \\
\hline
\end{tabular}

95\% CI 0.89 to $0.95 ; \mathrm{p}<0.001)$ were also statistically significant on univariable analysis (table 4). However, on multivariable analysis, only LV-EF emerged as an independent predictor (table 4, figure 3). Increasing amounts of fibrosis were associated with increased risk (figure 4) but this relationship did not hold after adjusting for LV-EF (table 4).

Table 2 Baseline cardiovascular magnetic resonance findings for the study cohort

\begin{tabular}{|c|c|c|c|c|}
\hline & $\begin{array}{l}\text { No } \\
\text { fibrosis }\end{array}$ & Fibrosis & $\begin{array}{l}\text { All } \\
\text { patients }\end{array}$ & $p$ Value \\
\hline CMR parameters- $\mathrm{n}(\%)$ & $240(33.8)$ & $471(66.2)$ & 711 & \\
\hline $\begin{array}{l}\text { Maximum end-diastolic } \\
\text { LV wall thickness-mm }\end{array}$ & $16.6 \pm 3.7$ & $20.8 \pm 5.2$ & $19.4 \pm 5.1$ & $<0.001$ \\
\hline LV-EDV index- $\mathrm{mL} / \mathrm{m}^{2}$ & $67.8 \pm 14.5$ & $69.8 \pm 16.2$ & $69.1 \pm 15.7$ & 0.123 \\
\hline LV-ESV index- $\mathrm{mL} / \mathrm{m}^{2}$ & $15.9 \pm 7.5$ & $19.3 \pm 10.3$ & $18.2 \pm 9.6$ & $<0.001$ \\
\hline LV EF-\% & $77.1 \pm 7.3$ & $73.3 \pm 9.5$ & $74.6 \pm 9.0$ & $<0.001$ \\
\hline$<50 \%$ & $1(0.4)$ & $11(2.3)$ & $12(1.7)$ & 0.021 \\
\hline $50-59 \%$ & $5(2.1)$ & $25(5.3)$ & $30(4.2)$ & \\
\hline$\geq 60 \%$ & $232(97.5)$ & $434(92.3)$ & $666(94.1)$ & \\
\hline $\mathrm{LV}$ mass index- $\mathrm{g} / \mathrm{m}^{2}$ & $88.7 \pm 25.1$ & $108.4 \pm 40.1$ & $101.9 \pm 37.0$ & $<0.001$ \\
\hline $\begin{array}{l}\text { Extent of LGE-\% } \\
\text { of LV mass }\end{array}$ & - & $9.5(9.9)$ & $6.3(9.2)$ & - \\
\hline $\begin{array}{l}\text { Median-\% of } \\
\text { LV Mass (IQR) }\end{array}$ & - & $5.9(2.2,13.3)$ & $2.2(0,9.1)$ & - \\
\hline
\end{tabular}

All values are mean \pm SD unless otherwise stated.

CMR, cardiovascular magnetic resonance; EDV, end-diastolic volume; ESV, end-systolic volume; LGE, late gadolinium enhancement.

\section{Secondary outcomes}

In total, $38(5.3 \%)$ of the 711 patients reached the principal secondary composite outcome measure of cardiovascular death $(25$ $(3.5 \%))$ or aborted SCD $(13(1.8 \%))$. Of the 25 cardiovascular deaths, $14(56 \%)$ were due to heart failure, 9 (36\%) to SCD and $2(8 \%)$ to thromboembolic stroke (table 3$)$. In the fibrosis group, $30(6.4 \%)$ patients versus $8(3.3 \%)$ in the no fibrosis group reached this outcome (HR for LGE: 2.24, 95\% CI 1.03 to 4.89; $\mathrm{p}=0.043$ ) (figure 2). Univariable analysis revealed that the amount of fibrosis was also a significant predictor (HR per $5 \%$ LGE: $1.26,95 \%$ CI 1.12 to $1.41 ; \mathrm{p}<0.001)$ together with LV-EF (figure 4), and non-sustained VT (see online supplementary table S1). However, after adjusting for the effects of LV-EF and nonsustained VT, neither the presence nor the amount of fibrosis remained statistically significant (see online supplementary table S1).

During follow-up, $55(7.7 \%)$ of the 711 patients reached the secondary end point of all-cause mortality. Of the 55 deaths, 25 (45.5\%) were due to cardiovascular causes. In contrast to the cardiovascular mortality composite, neither the presence nor the amount of fibrosis were significant univariable predictors (see online supplementary table S2, figure 2). After adjusting for the effects of LV-EF, NYHA class and previous sustained VT/VF, the amount of fibrosis again failed to reach statistical significance (see online supplementary table S2). LV-EF was the most significant independent predictor of death from any cause (figure 3).

For heart failure mortality, there were too few events to permit valid multivariable Cox regression analysis. However, on exploratory univariable analysis (table 3), similar to the primary and all-cause mortality end points, the amount but not the 

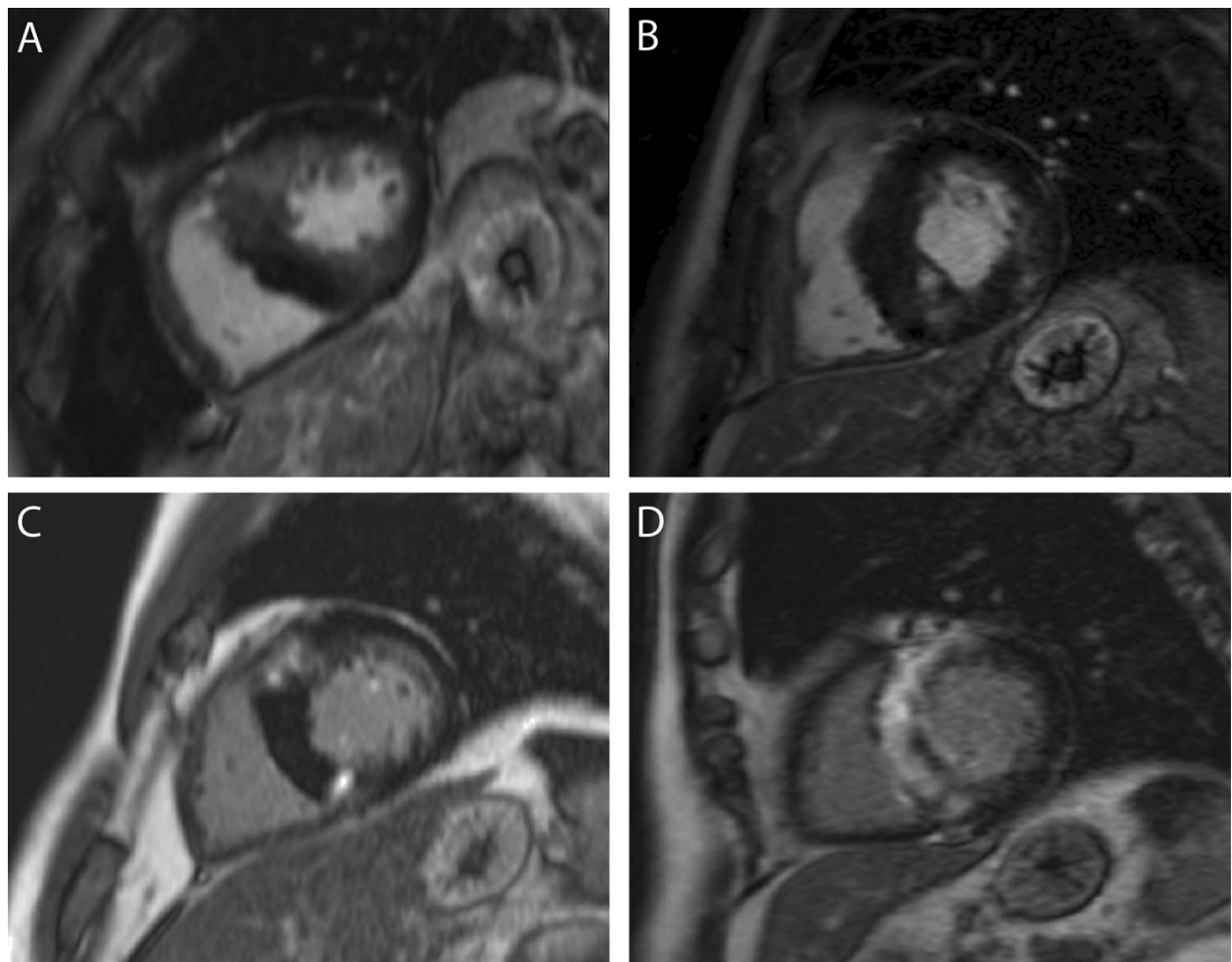

Figure 1 Representative examples of the patterns and spectrum of late gadolinium enhancement/fibrosis seen in hypertrophic cardiomyopathy. Diffuse fibrosis of the basal anteroseptum and inferoseptum (A); mixture of patchy and diffuse fibrosis of the inferoseptum and inferolateral walls (B); focal insertion point and mid-wall fibrosis (C); and extensive dense patchy fibrosis of the basal anteroseptum and inferoseptum (D).

presence of fibrosis was a significant univariable predictor of outcome (HR per 5\% LGE: 1.23 , 95\% CI 1.02 to 1.48 ; $\mathrm{p}<0.028$ ). No statistically significant interaction was detected between LV-EF and the amount of LGE for either primary end point (HR: 1.01, 95\% CI: 0.99 to $1.03, \mathrm{p}=0.303$ ) or either of the secondary end points (cardiovascular mortality HR: 1.00, $95 \%$ CI 0.99 to $1.02, p=0.680$; and all-cause mortality HR: $1.00,95 \%$ CI 0.99 to $1.01, \mathrm{p}=0.959$ ).

\section{DISCUSSION}

The pressing need to improve risk stratification in HCM has driven the search for better biomarkers that interrogate the underlying substrate responsible for heart failure and SCD. ${ }^{4}$ Myocardial fibrosis can be assessed non-invasively using LGE-CMR and is thought to play a key role in arrhythmogenesis and the development of contractile dysfunction, providing a strong pathophysiological rationale for evaluating it as a biomarker of risk in HCM. ${ }^{9}$ In the present study, CMR evidence of fibrosis was identified in two-thirds of patients and the amount was an important predictor of SCD or aborted SCD risk but did not provide independent prognostic information incremental to LV-EF. Similar results were obtained with respect to the principal secondary composite end point of cardiovascular mortality or aborted SCD.

The present findings add substantially to our earlier work, and two other previous smaller studies that have examined the prognostic utility of LGE-CMR in HCM. ${ }^{12-14}$ We previously found that the presence and the amount of fibrosis predicted adverse cardiovascular outcomes in 217 patients with HCM. ${ }^{12}$ In this earlier work, our primary composite end point

Table 3 Summary of outcomes for the whole cohort and stratified according to the presence/absence and the amount of fibrosis

\begin{tabular}{|c|c|c|c|c|c|c|c|c|c|c|c|}
\hline \multirow{3}{*}{$\begin{array}{l}\text { Outcome measure, } \mathbf{n}(\%) \\
\text { SCD or aborted SCD }\end{array}$} & \multirow{3}{*}{$\begin{array}{c}\text { No fibrosis } \\
240(33.8) \\
4(1.7)\end{array}$} & \multirow{3}{*}{$\begin{array}{l}\text { Fibrosis } \\
471(66.2) \\
18(3.8)\end{array}$} & \multirow{3}{*}{$\begin{array}{l}\text { All patients } \\
711 \\
22(3.1)\end{array}$} & \multicolumn{4}{|c|}{ Presence of fibrosis } & \multicolumn{4}{|c|}{ Amount of Fibrosis* } \\
\hline & & & & \multirow{2}{*}{$\begin{array}{l}\text { HR } \\
2.69\end{array}$} & \multicolumn{2}{|c|}{$95 \% \mathrm{Cl}$} & \multirow{2}{*}{$\begin{array}{l}p \text { Value } \\
0.073\end{array}$} & \multirow{2}{*}{$\begin{array}{l}\mathrm{HR} \\
1.24\end{array}$} & \multicolumn{2}{|c|}{$95 \% \mathrm{Cl}$} & \multirow{2}{*}{$\begin{array}{r}\mathrm{p} \text { Value } \\
0.007\end{array}$} \\
\hline & & & & & 0.91 & 7.97 & & & 1.06 & 1.45 & \\
\hline Cardiovascular death or aborted SCD & $8(3.3)$ & $30(6.4)$ & $38(5.3)$ & 2.24 & 1.03 & 4.89 & 0.043 & 1.26 & 1.12 & 1.41 & $<0.001$ \\
\hline Heart failure death & $4(1.7)$ & $10(2.1)$ & $14(2.0)$ & 1.49 & 0.47 & 4.78 & 0.498 & 1.23 & 1.02 & 1.48 & 0.028 \\
\hline SCD & $1(0.4)$ & $8(1.7)$ & $9(1.3)$ & 4.92 & 0.61 & 39.3 & 0.134 & 1.27 & 0.99 & 1.62 & 0.056 \\
\hline Aborted SCD & $3(1.3)$ & $10(2.1)$ & $13(1.8)$ & 1.96 & 0.54 & 7.13 & 0.307 & 1.22 & 1.00 & 1.50 & 0.054 \\
\hline Thromboembolic stroke & $0(0)$ & $2(0.4)$ & $2(0.3)$ & - & - & - & - & - & - & - & - \\
\hline All-cause mortality & $16(6.7)$ & $39(8.3)$ & $55(7.7)$ & 1.44 & 0.81 & 2.58 & 0.218 & 1.11 & 0.99 & 1.24 & 0.068 \\
\hline
\end{tabular}



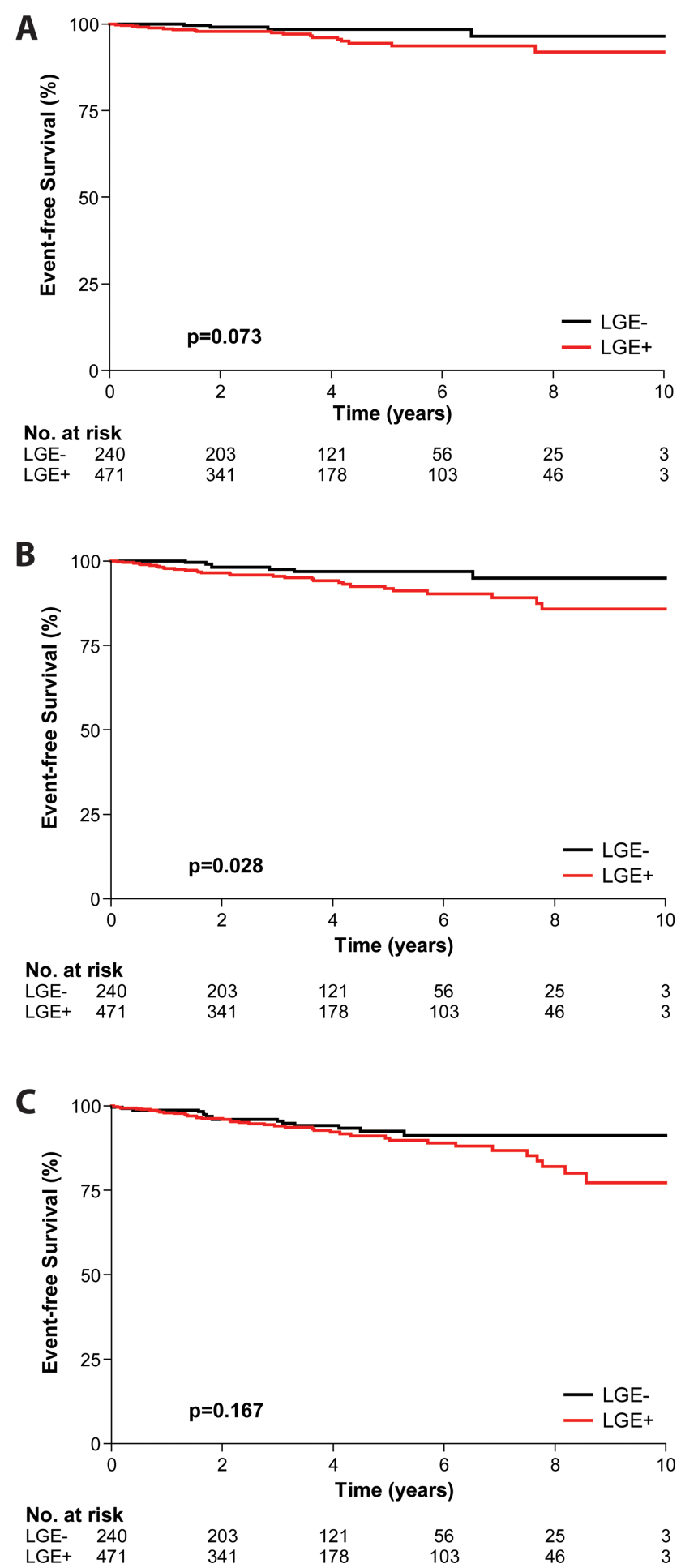

Figure 2 Kaplan-Meier estimates of event-free survival for the primary outcome measure of sudden cardiac death or aborted sudden cardiac death (A); the principal secondary end point of cardiovascular mortality or aborted sudden cardiac death (B); and all-cause mortality (C) stratified according to the presence and absence of fibrosis. LGE, late gadolinium enhancement.

incorporated unplanned cardiovascular hospitalisation which differed significantly between patients with and without fibrosis. Furthermore, there were only nine cardiovascular deaths and we were therefore not sufficiently powered to identify a difference in either cardiovascular mortality or SCD between the two groups. In the present much larger study with longer follow-up, there were 22 SCDs or aborted SCDs and 38 cardiovascular deaths or aborted SCDs in total, allowing us to make more robust inferences on the prognostic value of fibrosis.

Bruder $e a^{13}$ conducted the only other published prospective LGE-CMR study to date using cardiovascular mortality as an end point. They were able to demonstrate that fibrosis was a statistically significant univariable predictor of outcome, but with only a total of 16 events, similar to our own previous work, their study was not sufficiently powered to demonstrate the independent prognostic significance of fibrosis with respect to SCD or cardiovascular mortality over and above potential confounders. ${ }^{9}$ In particular, as in the present study, fibrosis was highly associated with significant hypertrophy, defined in terms of maximal LV wall thickness and indexed LV mass. There was also a significant excess of patients with non-sustained VT in their fibrosis group relative to those without fibrosis. The latter association has also been noted in numerous earlier studies. ${ }^{8} 1112152223$

In the only other published prospective LGE-CMR study, Maron et al ${ }^{14}$ evaluated the clinical significance of fibrosis in 202 patients with HCM using a composite primary end point of cardiovascular death or progressive heart failure, which was defined as a change in NYHA class. Despite the use of this broad end point, and the inclusion of antitachycardia pacing as a SCD-equivalent, only 11 adverse cardiovascular events occurred over a mean follow-up of 1.9 years. Their event rate was therefore insufficient to identify either the presence or the amount of fibrosis as even an univariable predictor of outcome. ${ }^{9}$ However, in keeping with the present study, a statistically significant inverse association was found between LV systolic function as measured by LV-EF and the presence and amount of fibrosis. ${ }^{14}$ The latter finding is also in accordance with the results of Rubinshtein et al. $^{15}$ Their retrospective cohort study also identified the presence of fibrosis as a significant univariable predictor of SCD or appropriate ICD discharge. However, as only eight events occurred among their 424 patients after a mean of 3.6 years follow-up, in common with previous studies, ${ }^{13} 14$ they were underpowered to adjust for statistically significant differences in baseline characteristics between those with and without fibrosis. ${ }^{15}$

In the present study, LV-EF emerged as the most powerful independent predictor of SCD risk as well as cardiovascular and allcause mortality. Although widely used to guide the management of patients with ischaemic heart disease and non-ischaemic dilated cardiomyopathy, ${ }^{25} 26$ the assessment of LV systolic function is not currently emphasised in guidelines for the risk stratification of HCM. ${ }^{17}$ In the largest series to date, Harris et al examined the prevalence and significance of adverse LV remodelling in HCM. ${ }^{27}$ Of their retrospectively identified cohort of 1259 consecutive patients with HCM, 44 (3.5\%) had evidence of LV systolic dysfunction defined by echocardiography as $\mathrm{LV}-\mathrm{EF}<50 \%$ at rest. In our cohort, the prevalence of LV dysfunction defined using an equivalent CMR threshold of $\mathrm{LV}-\mathrm{EF} \leq 55 \%$ was similar in $23(3.2 \%)$ of 711 patients. However, of these, 5 (21.7\%) reached the primary end point of SCD or aborted SCD, and $9(39.1 \%)$ reached the principal secondary cardiovascular composite outcome in comparison with two-thirds of patients in the Harris cohort, although their higher incidence of adverse cardiovascular events may reflect their longer follow-up duration. Of note, $9(81 \%)$ of a subgroup of 11 of their end-stage patients had evidence of extensive fibrosis on CMR or at autopsy. In the present study, 21 of 23 patients (91\%) with LV dysfunction had CMR evidence of fibrosis. The superior predictive value of LV-EF over and above fibrosis may reflect the fact that it provides a better synthesis of the various ventricular effects of HCM, 
Table 4 Results of univariable and multivariable analyses of the predictors of sudden cardiac death or aborted sudden cardiac death

\begin{tabular}{|c|c|c|c|c|}
\hline Univariable Analysis & Hazard ratio & $95 \% \mathrm{Cl}$ & & $p$ Value \\
\hline Male & 1.42 & 0.52 & 3.84 & 0.493 \\
\hline New York Heart Association functional class & 1.02 & 0.54 & 1.93 & 0.951 \\
\hline Sustained ventricular tachycardia/fibrillation & 7.76 & 1.79 & 33.57 & 0.006 \\
\hline Family history of sudden cardiac death & 0.75 & 0.17 & 3.21 & 0.698 \\
\hline LV wall thickness $\geq 30 \mathrm{~mm}$ & 1.62 & 0.38 & 6.98 & 0.516 \\
\hline LV outflow tract obstruction $\geq 30 \mathrm{~mm} \mathrm{Hg}$ & 0.50 & 0.17 & 1.47 & 0.207 \\
\hline Non-sustained ventricular tachycardia & 1.71 & 0.40 & 7.38 & 0.472 \\
\hline Unexplained syncope & 0.84 & 0.19 & 3.59 & 0.809 \\
\hline Number of risk factors & 1.00 & 0.55 & 1.80 & 0.995 \\
\hline Maximum LV end-diastolic wall thickness (mm) & 1.01 & 0.93 & 1.09 & 0.845 \\
\hline Indexed LV end-diastolic volume (per $10 \mathrm{ml} / \mathrm{m}^{2}$ ) & 1.17 & 0.89 & 1.54 & 0.257 \\
\hline Indexed LV end-systolic volume (per $10 \mathrm{ml} / \mathrm{m}^{2}$ ) & 1.88 & 1.45 & 2.45 & $<0.001$ \\
\hline LV EF (\%) & 0.92 & 0.89 & 0.95 & $<0.001$ \\
\hline LV mass index (per $10 \mathrm{~g} / \mathrm{m}^{2}$ ) & 1.07 & 0.99 & 1.17 & 0.104 \\
\hline Presence of fibrosis & 2.69 & 0.91 & 7.97 & 0.073 \\
\hline Amount of fibrosis (per $5 \%$ of LV mass) & 1.24 & 1.06 & 1.45 & 0.007 \\
\hline \multicolumn{5}{|l|}{ Multivariable analysis } \\
\hline \multicolumn{5}{|l|}{ Presence of fibrosis as a candidate } \\
\hline LV EF (\%) & 0.92 & 0.89 & 0.95 & $<0.001$ \\
\hline Presence of fibrosis & 2.98 & 0.66 & 13.44 & 0.155 \\
\hline \multicolumn{5}{|l|}{ Amount of fibrosis as a candidate } \\
\hline LV EF (\%) & 0.92 & 0.89 & 0.95 & $<0.001$ \\
\hline Amount of fibrosis (per $5 \%$ of LV mass) & 1.10 & 0.92 & 1.31 & 0.299 \\
\hline
\end{tabular}

which include fibrosis and genetically mediated contractile dysfunction and energetic abnormalities. ${ }^{28}$

Although the poor prognosis of end-stage or incipient end-stage disease is recognised, LV dysfunction is not sufficiently emphasised in current clinical guidelines for risk stratification. ${ }^{17} 29$ As a consequence of LV hypertrophy, LV end-diastolic volumes tend to be small in HCM, resulting in large fractional volume changes in systole. Thus, even an apparently borderline EF may be a harbinger of incipient adverse outcomes analogous to the situation of LV volume overload due to chronic mitral regurgitation.

\section{Limitations}

Our study was conducted at a single tertiary centre. While this facilitated the use of a standardised scanning and follow-up protocol, it also incurred the possibility of selection bias. However, referrals to our centre are drawn from an extensive network of district general hospitals spanning the entire southeast and the wider UK, mitigating against this possibility. Although the event rates observed in the present study were considerably higher than those found in community-based cohorts, ${ }^{1}{ }^{30}$ they are comparable with those seen in other studies of patients who have come to clinical attention. ${ }^{13-15}$ Our data are therefore representative of patients encountered by cardiologists in clinical practice. Indeed, high-risk patients may have been inadvertently selected out from our cohort. For instance, those with an ICD at baseline were necessarily excluded as this represents a contraindication to CMR. Similarly, patients with previous gradient reduction therapy were also excluded as this introduces confounding iatrogenic scar, potentially lowering the event rate observed.

The amount of LGE was a statistically significant univariate predictor of the primary and principal secondary end points. This implies by definition that our study was adequately powered to detect a statistically significant difference in outcome based on the amount of LGE. However, on limited further modelling, the amount of LGE did not emerge as an independent risk factor incremental to other factors. While we cannot exclude the possibility that a significantly larger sample size and/or longer duration of follow-up would be able to tease out a separate independent effect, we believe that this implies that the effect size that we would be trying to detect is very small and therefore the positive predictive value of the amount of LGE over and above existing markers is likely to be very limited.

Although 49 patients were lost to follow-up, we can confirm from death certificate records that none of them died within the UK. Nevertheless, we cannot exclude the possibility that these patients may have emigrated overseas or while within the UK, received an ICD which was then subsequently liable to discharge. However, generously assuming a device implantation rate of $10 \%$, only $\sim$ five patients would have received an ICD. With an annual discharge rate for primary prevention of $3.6 \%$ per annum with a cumulative 5 -year probability of $17 \%,{ }^{2}$ only $\sim$ one patient is likely to have received an appropriate shock over 5 years (our median follow-up was 3.5 years). Therefore, it is unlikely that statistically significant events were not captured that would have altered our data interpretation. Furthermore, among the 49 patients lost to follow-up, 24 (49.0\%) had no fibrosis versus $240(33.8 \%)$ of the 711 patients with full follow-up $(p=0.0426)$. Thus, significantly more patients without LGE were lost to follow-up, making it even more unlikely that undetected events would have altered our assessment.

Finally, the LGE-CMR technique exploits relative differences in signal intensity between fibrotic and ostensibly normal myocardium. The full-width at half-maximum technique has been shown to be the most reproducible quantification method. ${ }^{20}$ 
A

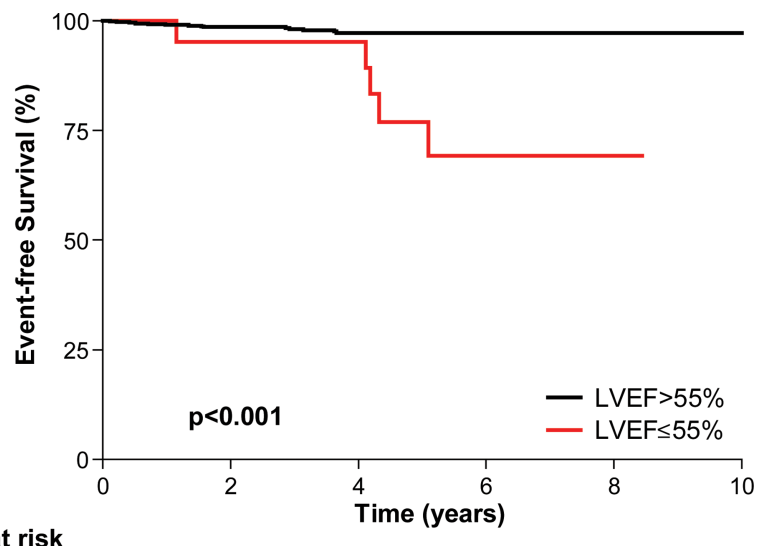

No. at risk

$\begin{array}{lcccccc}\text { LVEF }>55 \% & 685 & 524 & 281 & 152 & 70 & 6 \\ \text { LVEF } \leq 55 \% & 23 & 18 & 16 & 5 & 1 & 0\end{array}$

B

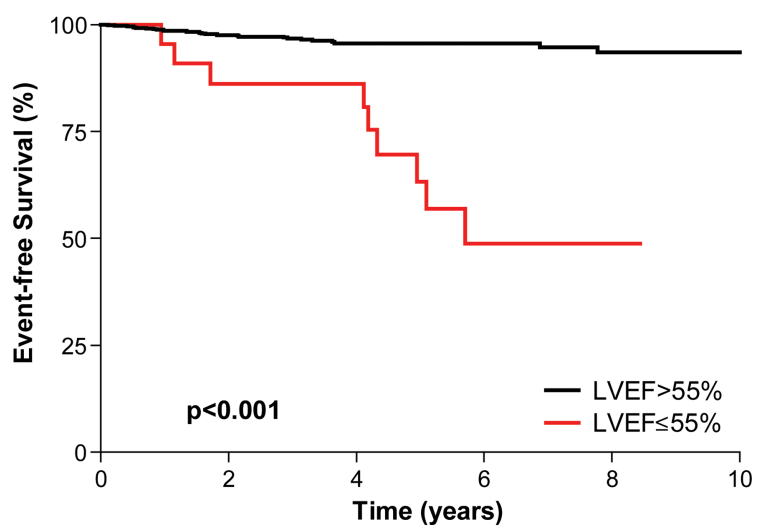

No. at risk

$\begin{array}{lcccccc}\text { LVEF }>55 \% & 685 & 524 & 281 & 152 & 70 & 6 \\ \text { LVEF } \leq 55 \% & 23 & 18 & 16 & 5 & 1 & 0\end{array}$

\section{C}

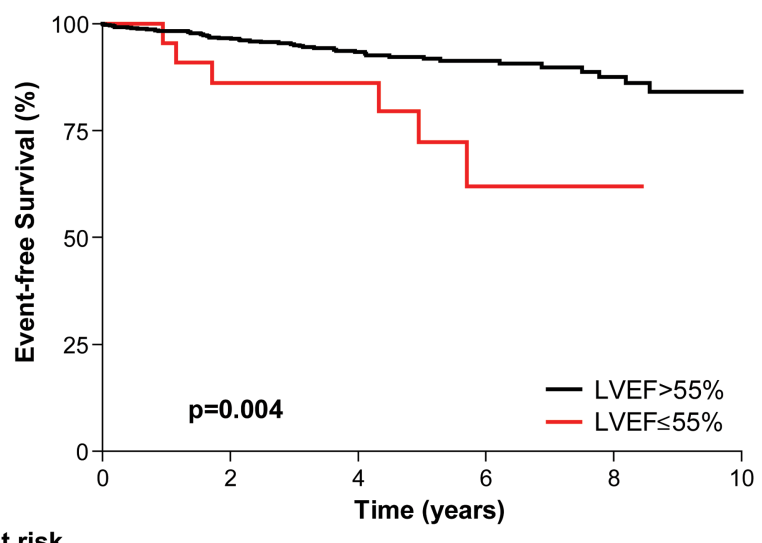

No. at risk

$\begin{array}{llccccc}\text { LVEF }>55 \% & 685 & 524 & 281 & 152 & 70 & 6\end{array}$

Figure 3 Kaplan-Meier estimates of event-free survival for the primary outcome measure of sudden cardiac death or aborted sudden cardiac death $(A)$; the principal secondary end point of cardiovascular mortality or aborted sudden cardiac death (B); and all-cause mortality (C) stratified according to LVEF.

Nevertheless, there is no widespread consensus on the best quantification strategy to use and a paucity of histological validation for the LGE technique in the setting of cardiomyopathy. ${ }^{11} 1213$

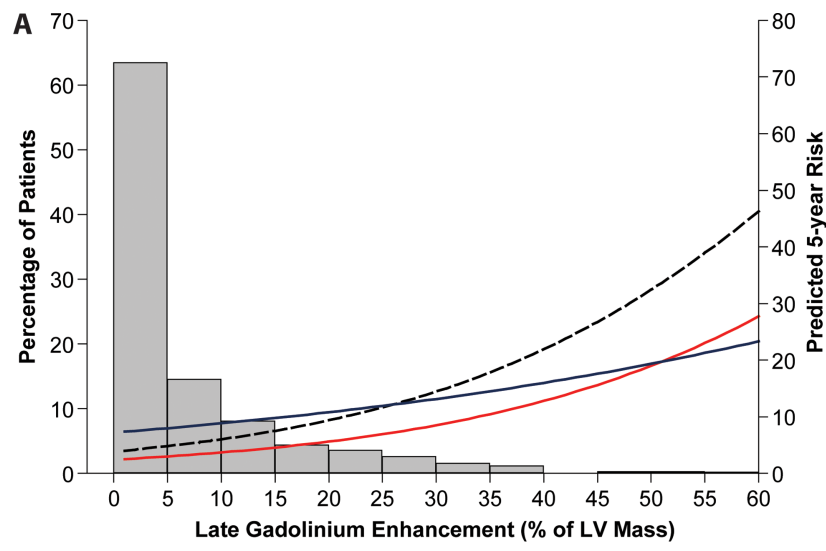

- SCD or ASCD

-- CV Death or ASCD - AC Mortality

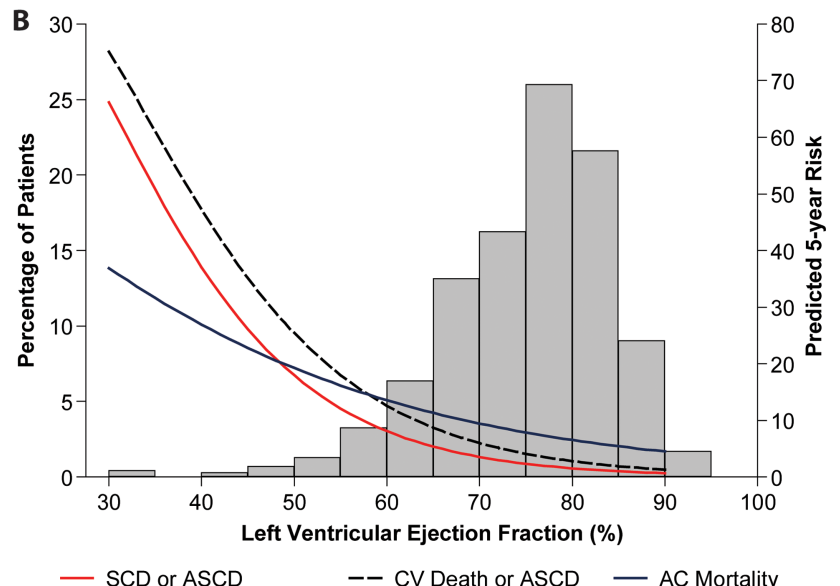

Figure 4 Predicted 5-year risk of reaching the study outcome measures according to amount of fibrosis (A) and LVEF (B). ASCD, aborted sudden cardiac death; CV, cardiovascular; AC, all-cause; SCD, sudden cardiac death.

\section{Key messages}

What is known on this subject?

Multiple small retrospective and prospective cohort studies using composite outcome measures have suggested that the presence and amount of myocardial fibrosis, as detected by late gadolinium enhancement (LGE)-cardiovascular magnetic resonance (CMR), are associated with adverse outcomes in hypertrophic cardiomyopathy (HCM). However, none of these studies were of sufficient size and/or duration to be able to address the prognostic significance of myocardial fibrosis with respect to sudden cardiac death $(\mathrm{SCD})$ risk, leaving the role of LGE-CMR in HCM risk stratification unresolved.

\section{What might this study add?}

This is the first study to definitively confirm an association between the amount of fibrosis, but not its mere presence, and SCD risk. However, after adjusting for other factors such as LV-EF, the amount of fibrosis did not retain independent significance.

How might this impact on clinical practice?

The amount of myocardial fibrosis is an important predictor of SCD risk in $\mathrm{HCM}$, but may not provide information incremental to other factors. In contrast, LV dysfunction emerged as an independent risk factor and should be given greater emphasis in clinical guidelines as a harbinger of increased SCD risk. 


\section{CONCLUSIONS}

The amount of myocardial fibrosis is an important predictor of outcome in HCM but may not provide information incremental to LV-EF. The latter is an important prognosticator for outcome in HCM, which is underemphasised in current guidelines. Further work is required to elucidate the interrelationship between myocardial fibrosis and other markers of risk in HCM.

Acknowledgements The authors are grateful to the staff of the CMR Unit and the Cardiovascular BRU, Royal Brompton Hospital for their support with this work and the medical and administrative staff at referring centres and primary care centres who assisted with data collection.

Contributors SKP and DJP conceived the study and were responsible for study design. WJM, JCM, MRC, MR, ROH and PE advised on study design. TFI, AJ, AG, $A M, S R, T E C, B D, J K, F D A, R W, A V, C S, S A C$ and ROH were involved in data gathering, analysis, interpretation as well as drafting of the manuscript. MR was responsible for all data and statistical analysis. TFI drafted the initial manuscript. All authors were involved in the critical revision of the manuscript for important intellectual content.

Funding This work was supported by the National Institute of Health Research Cardiovascular Biomedical Research Unit at Royal Brompton and Harefield NHS Foundation Trust and Imperial College. TFI was funded by the British Heart Foundation. AJ was supported by the National Health and Medical Research Council of Australia and the Victor Chang Cardiac Research Institute. AG received grant support from CORDA, the research charity, and the Rosetrees Trust.

Competing interests SKP has received honoraria from Bayer-Schering. DJP is a consultant to Siemens and a director of Cardiovascular Imaging Solutions. Royal Brompton Hospital has a research collaboration agreement with Siemens. MRC has received speaker fees from Roche Diagnostics and Pfizer; grants from Medtronic and ResMed; and is a consultant to Novartis, Medtronic, St Jude Medical, Boston Scientific, GE Medical and Servier. None of the other authors report any disclosures relevant to this work.

Ethics approval Royal Brompton and Harefield NHS Trust Ethics Committee.

Provenance and peer review Not commissioned; externally peer reviewed.

\section{REFERENCES}

1 Maron BJ. Hypertrophic cardiomyopathy: a systematic review. JAMA 2002;287:1308-20.

2 Maron BJ, Spirito P, Shen WK, et al. Implantable cardioverter-defibrillators and prevention of sudden cardiac death in hypertrophic cardiomyopathy. JAMA 2007;298:405-12.

3 O'Mahony C, Lambiase PD, Quarta G, et al. The long-term survival and the risks and benefits of implantable cardioverter defibrillators in patients with hypertrophic cardiomyopathy. Heart 2012;98:116-25.

4 Maron BJ. Contemporary insights and strategies for risk stratification and prevention of sudden death in hypertrophic cardiomyopathy. Circulation 2010;121:445-56.

5 Elliott PM, Gimeno Blanes JR, Mahon NG, et al. Relation between severity of left-ventricular hypertrophy and prognosis in patients with hypertrophic cardiomyopathy. Lancet 2001;357:420-4.

6 O'Mahony C, Tome-Esteban M, Lambiase PD, et al. A validation study of the 2003 American College of Cardiology/European Society of Cardiology and 2011 American College of Cardiology Foundation/American Heart Association risk stratification and treatment algorithms for sudden cardiac death in patients with hypertrophic cardiomyopathy. Heart 2013;99:534-41.

7 Elliott P, McKenna WJ. Hypertrophic cardiomyopathy. Lancet 2004;363:1881-91.

8 Moon JC, McKenna WJ, McCrohon JA, et al. Toward clinical risk assessment in hypertrophic cardiomyopathy with gadolinium cardiovascular magnetic resonance. J Am Coll Cardiol 2003:41:1561-7.

9 Ismail TF, Prasad SK, Pennell DJ. Prognostic importance of late gadolinium enhancement cardiovascular magnetic resonance in cardiomyopathy. Heart 2012;98:438-42.

10 Green JJ, Berger JS, Kramer CM, et al. Prognostic value of late gadolinium enhancement in clinical outcomes for hypertrophic cardiomyopathy. JACC Cardiovasc Imaging 2012;5:370-7.

11 Adabag AS, Maron BJ, Appelbaum E, et al. Occurrence and frequency of arrhythmias in hypertrophic cardiomyopathy in relation to delayed enhancement on cardiovascular magnetic resonance. J Am Coll Cardiol 2008;51:1369-74.
12 O'Hanlon R, Grasso A, Roughton M, et al. Prognostic significance of myocardial fibrosis in hypertrophic cardiomyopathy. J Am Coll Cardiol 2010;56:867-74.

13 Bruder 0, Wagner A, Jensen CJ, et al. Myocardial scar visualized by cardiovascular magnetic resonance imaging predicts major adverse events in patients with hypertrophic cardiomyopathy. J Am Coll Cardiol 2010;56:875-87.

14 Maron MS, Appelbaum E, Harrigan CJ, et al. Clinical profile and significance of delayed enhancement in hypertrophic cardiomyopathy. Circ Heart Fail 2008:1:184-91.

15 Rubinshtein R, Glockner JF, Ommen SR, et al. Characteristics and clinical significance of late gadolinium enhancement by contrast-enhanced magnetic resonance imaging in patients with hypertrophic cardiomyopathy. Circ Heart Fail 2010:3:51-8

16 Salerno M, Kramer CM. Prognosis in hypertrophic cardiomyopathy with contrast-enhanced cardiac magnetic resonance: the future looks bright. J Am Coll Cardiol 2010;56:888-9.

17 Gersh BJ, Maron BJ, Bonow RO, et al. 2011 ACCF/AHA Guideline for the Diagnosis and Treatment of Hypertrophic Cardiomyopathy: a report of the American College of Cardiology Foundation/American Heart Association Task Force on Practice Guidelines. Developed in collaboration with the American Association for Thoracic Surgery, American Society of Echocardiography, American Society of Nuclear Cardiology, Heart Failure Society of America, Heart Rhythm Society, Society for Cardiovascular Angiography and Interventions, and Society of Thoracic Surgeons. J Am Coll Cardiol 2011;58:e212-60.

18 Kramer CM, Barkhausen J, Flamm SD, et al. Standardized cardiovascular magnetic resonance imaging (CMR) protocols, society for cardiovascular magnetic resonance: board of trustees task force on standardized protocols. J Cardiovasc Magn Reson 2008; 10:35.

19 Simonetti OP, Kim RJ, Fieno DS, et al. An improved MR imaging technique for the visualization of myocardial infarction. Radiology 2001;218:215-23.

20 Flett AS, Hasleton J, Cook C, et al. Evaluation of techniques for the quantification of myocardial scar of differing etiology using cardiac magnetic resonance. JACC Cardiovasc Imaging 2011;4:150-6.

21 Spiewak M, Malek LA, Misko J, et al. Comparison of different quantification methods of late gadolinium enhancement in patients with hypertrophic cardiomyopathy. Eur J Radiol 2010;74:e149-53.

22 Moon JC, Mogensen J, Elliott PM, et al. Myocardial late gadolinium enhancement cardiovascular magnetic resonance in hypertrophic cardiomyopathy caused by mutations in troponin I. Heart 2005;91:1036-40.

23 Kwon DH, Smedira NG, Rodriguez ER, et al. Cardiac magnetic resonance detection of myocardial scarring in hypertrophic cardiomyopathy: correlation with histopathology and prevalence of ventricular tachycardia. J Am Coll Cardiol 2009;54:242-9.

24 Greenberg H, Case RB, Moss AJ, et al. Analysis of mortality events in the Multicenter Automatic Defibrillator Implantation Trial (MADIT-II). J Am Coll Cardiol 2004;43:1459-65.

25 Goldberger JJ, Cain ME, Hohnloser SH, et al. American Heart Association/American College of Cardiology Foundation/Heart Rhythm Society Scientific Statement on Noninvasive Risk Stratification Techniques for Identifying Patients at Risk for Sudden Cardiac Death. A scientific statement from the American Heart Association Council on Clinical Cardiology Committee on Electrocardiography and Arrhythmias and Council on Epidemiology and Prevention. J Am Coll Cardiol 2008;52:1179-99.

26 Zipes DP, Camm AJ, Borggrefe M, et al. ACC/AHA/ESC 2006 guidelines for management of patients with ventricular arrhythmias and the prevention of sudden cardiac death--executive summary: A report of the American College of Cardiology/ American Heart Association Task Force and the European Society of Cardiology Committee for Practice Guidelines (Writing Committee to Develop Guidelines for Management of Patients with Ventricular Arrhythmias and the Prevention of Sudden Cardiac Death) Developed in collaboration with the European Heart Rhythm Association and the Heart Rhythm Society. Eur Heart J 2006;27:2099-140.

27 Harris KM, Spirito P, Maron MS, et al. Prevalence, clinical profile, and significance of left ventricular remodeling in the end-stage phase of hypertrophic cardiomyopathy. Circulation 2006;114:216-25.

28 Ashrafian H, McKenna WJ, Watkins H. Disease pathways and novel therapeutic targets in hypertrophic cardiomyopathy. Circ Res 2011;109:86-96.

29 Maron BJ, McKenna WJ, Danielson GK, et al. American College of Cardiology/ European Society of Cardiology Clinical Expert Consensus Document on Hypertrophic Cardiomyopathy. A report of the American College of Cardiology Foundation Task Force on Clinical Expert Consensus Documents and the European Society of Cardiology Committee for Practice Guidelines. Eur Heart J 2003:24:1965-91.

30 Cecchi F, Olivotto I, Montereggi A, et al. Hypertrophic cardiomyopathy in Tuscany: clinical course and outcome in an unselected regional population. J Am Coll Cardiol 1995;26:1529-36. 